\title{
Research Paper: Comparison of Knee Kinematic Factors Between Ball and Rocket Athletes in Pre- dictable and Unpredictable Cutting Maneuvers
}

\author{
Elham Hosseini $^{1^{*}}$ (D), Abdolhamid Daneshjoo ${ }^{1}$ iD, Mansour Sahebozamani ${ }^{1}$ (D)
}

1. Department of Sports Injuries and Corrective Exercises, Faculty of Sports Sciences, Shahid Bahonar University of Kerman, Kerman, Iran.

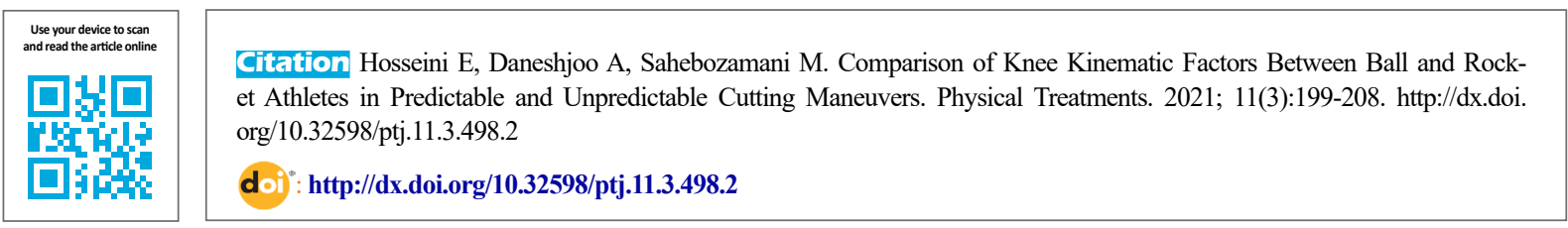

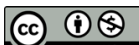

Article info:

Received: 17 Jan 2021

Accepted: 02 Mar 2021

Available Online: 01 Jul 2021

Keywords:

Knee flexion, Knee

valgus, Knee injury, Side-

Step cutting
A B S T R A C T

Purpose: Anterior cruciate ligament injury is one of the most severe knee injuries that often has a non-contact mechanism and follows an incorrect knee joint alignment. The present study aimed to compare the effect of predictable and unpredictable cutting direction on knee joint kinematics in ball and racket athletes.

Methods: The present study was quasi-experimental, and the subjects included 48 female adolescent athletes from badminton $(n=12)$, table tennis $(n=12)$, basketball $(n=12)$, and handball $(n=12)$. After the initial evaluations, the cutting maneuver was performed in predictable and unpredictable ways and recorded by a three-dimension motion analysis. Also, 1-way ANOVA and post hoc tests were used to compare the kinematic parameters of the knee between the ball and racket athletes.

Results: The results of the statistical test showed a significant difference in the kinematic parameters of knee flexion $(\mathrm{P}=0.003)$, knee valgus $(\mathrm{P}=0.001)$, and tibia rotation $(\mathrm{P}=0.001)$ between the ball (basketball and handball) and racket athletes (badminton and table tennis) in the predictable cutting. But in the unpredictable cutting, there were no significant differences in the kinematic parameters of the knee flexion $(\mathrm{P}=0.86)$, knee valgus $(\mathrm{P}=0.56)$, and tibia rotation $(\mathrm{P}=0.18)$.

Conclusion: The results indicated that ball athletes showed an increased kinematic risk factor such as decreased knee flexion, increased knee valgus, and tibia rotation more than rocket athletes. Therefore, injury prevention programs are more critical in ball athletes.

\section{* Corresponding Author:}

Elham Hosseini, MSc.

Address: Department of Sports Injuries and Corrective Exercises, Faculty of Sports Sciences, Shahid Bahonar University of Kerman, Kerman, Iran

Phone: +98 (34) 33120607

E-mail: hosseinelham7400@sport.uk.ac.ir 


\section{Highlights}

- There are more dangerous kinematic parameters in the group of ball sports such as basketball or handball.

- The response will significantly effect on the athlete's performance

\section{Plain Language Summary}

Athletes in unpredictability sport environments need to quickly choose movement strategies in response to changes of those around them. This response will significantly effect on the athlete's performance and the joint position among sport maneuvers. The cutting side maneuver performs suddenly and frequently in ball and rocket sports and these factors can increase the risk of injury especially non-contact injury in knee joint. because performing cutting side maneuvers in sports conditions with unpredictable factors such as the direction of the ball or pass or even the presence of the opponents or spectators can lead to technical errors and injury. Adolescent girls are more exposed to the risk of injury, and this injury can have devastating effects on a persons life. So, it's suggested that to prevent sports injuries and psychological and physical consequences resulting from it, and to promote the level of competition, the issue of predictability and correct training of techniques in designing exercise for this age group, especially in ball fields, receive more attention. additionally we can reduced the prevalence of non-contact injuries especially in knee joint injuries, by increased athletes awareness and correct performance.

\section{Introduction}

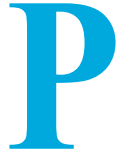

redictability in difficult sports conditions, especially at high-speed competitions, is essential for preparing appropriate movement responses to opponent behaviors so that the professional athlete could correctly guess the opponent's movement behaviors in the same initial phase of the movement and show a proper reaction. In general, predictability is a strategy to reduce processing stages time naturally used in response to an unpredictable stimulus [1]. Athletes in unpredictable sport environments need to quickly choose movement strategies in response to changes of those around them. This response will significantly affect the athlete's performance and the joint position among sports maneuvers [2]. In other words, in unpredictable conditions, people with less predictability cannot correct joint position, such as maximum joint angles and joint displacement in the initial contact. Consequently, unpredictable landing and cutting maneuvers can increase the risk of non-contact knee injuries [3].

Cutting side maneuver is a movement in the closed chain used by athletes of various sports fields to change the direction of movement [4]. The Anterior Cruciate Ligament (ACL) is often injured when the lower limb acceleration decreases in dynamic movements, and these movements usually include cutting side maneuvers. It should be mentioned that the cutting side maneuver performs suddenly and frequently in ball and racket sports and increase the risk of injury by placing a significant eccentric force on the lower limb [5]. The cutting side maneuvers performing by a high-speed and sudden change of direction can be a risk factor for ACL injury [6].

According to the literature, the cutting maneuver performed in sports fields is entirely different from that performed in laboratory conditions. Because performing cutting side maneuvers in sports conditions with unpredictable factors such as the direction of the ball or pass or the presence of the opponents and spectators can lead to technical errors and more changes in the kinematic parameters [7]. The cutting side maneuver has been identified as an essential risk factor concerning non-contact ACL injury, especially in female athletes. It has also been reported that women are 2 to 10 times more vulnerable to knee injuries than men [4]. ACL rupture is one of the most severe injuries of the knee joint. According to literature, the mechanism of this injury is often non-contact type, so biomechanical risk factors play an essential role in its occurrence [8].

In this regard, Yum et al. have shown that during an unpredicted cutting side concurrent changing of the kinematic parameters of the knee joint, the imposed loads are greater than those applied during the predictable maneuver. This condition can lead to ACL injury [2], even though Nouri et al. argued that professional athletes could better control the correct movement and appropriate position of the joints in unpredictable conditions and perform safe sports maneuvers such as cutting 
and reducing acceleration [1]. But Patrick et al. reported that the athletes of racket fields have better perceptual abilities such as reaction time and predictability than the athletes of ball fields [9]. These results could be a reason for the increased rate of ACL injury in ball fields such as basketball and handball [8]. In addition, several studies have compared athletes in different fields in terms of predictability; these studies have shown that regular exercise improves the ability to predict, and athletes in the different fields differ significantly in the amount of predictability [10-13]. Also, Omar et al. investigated the visual anticipation time differences between athletes in open and closed skills sports. They showed that participation in open skills sports such as badminton, tennis, basketball, and handball could improve an athlete's ability to predict [14]. Despite these research studies, no study has been found on the effects of cognitive abilities on kinematic parameters joint during sports maneuver between ball and racket sports.

Generally, the prevention of knee injuries, especially ACL injury in various sports, depends on recognizing possible risk factors and specifying prevention methods. In this regard, many studies have examined the potential risk factors for ACL injury. However, there is a scientific gap regarding the risk factors for ACL injury. Because the risk factors for ACL injury are multifactor, most studies have examined only one risk factor separately, and similar conditions of the sports environment have less been investigated $[15,16]$. As stated, predictability and decision-making are effective in improving kinematic indicators and preventing athletes' injury [17, 18]. Regarding the lack of studies in the field of different sports fields and the rate of predictability in athletes of various sports fields [14], the present study investigated the effect of prediction ability on the knee kinematic parameters during cutting side movement between the ball and racket athletes.

\section{Materials and Methods}

The present research of quasi-experimental type was performed in the laboratory of the Faculty of Sports Sciences of Shahid Bahonar University of Kerman Province, Iran. The statistical population consisted of all female adolescent ball and racket athletes in Kerman City, Iran. The sample size was determined 49 athletes using the GPower program and considering the statistical power of 0.9 and a significance level of 0.05 . The effect size of each variable was tested using partial eta squared $(\eta)(0.01=$ small effect, $0.06=$ medium effect, and 0.14=large effect) [19]. For this purpose, 48 female adolescent athletes in Kerman were selected randomly and participated in the present research in two groups of ball sports $=24$ athletes (including handball $=12$ athletes, basketball $=12$ athletes) and racket sports $=24$ athletes (including badminton $=12$ athletes, table tennis $=12$ athletes). The inclusion criteria were having at least 3 years of experience in regular sports exercises (at least 3 sessions per week), being between 13 and 16 years old, having normal body mass index (BMI), and lacking any injury and lower limb surgery in the past 6 months [4]. Also, people who had a history of lower limb injury and surgery in the past 6 months, had severe physical activity 24 hours before the test, or participated in ACL injury prevention programs were excluded from the research [6]. It should be mentioned that the present research was approved by the Ethics Committee in Research of Shahid Bahonar University of Kerman (Code: IR.UK.VETMED.RED.1398.022).

Before the test, the subjects were informed about the importance and purpose of the research, the procedure, and the test implementation. Then, the subjects gave their consent to participate in the research project and the athletes' health questionnaire. According to the researcher's timing, subjects in each group attended the laboratory on two different days that at least 24 hours had passed from their strenuous physical activity. During the testing sessions, clinical examinations (weight and height measurement), marking and warm-up protocol (10 minutes of soft running, stretching, and strength movements specific to the lower limb), tests for determining the dominance leg, a sprint of $10 \mathrm{~m}$, the agility t-test [18] and cutting side protocol and kinematic sampling were performed. It should be mentioned that during the testing stages, we tried that all controllable conditions, including brightness, temperature, noise, and testing stages were the same for all subjects to measure as far as possible the effect of the independent variable. According to the findings of previous research, while doing the cutting side movement and in the progress stage in the change of direction movement, the body accelerates in the opposite direction of movement, and the non-dominant leg encounters a decrease in flexion rate and an increase in valgus rate and tibia rotation [20, 21]. Therefore, the probability of injury in the non-dominant leg is higher, and for this reason, in the present research, the kinematic data of the non-dominant leg (support leg) were examined.

Afterward, the subjects were asked to perform the test with minimal clothing to prevent the movement of markers and to evaluate the kinematic parameters of the knee accurately. Marking was done manually and on both limbs in a three-dimensional manner, so that on 
each shin and hip limbs, three markers by cluster method (6 per leg), and two markers on both sides of the knee joint line (16 markers in total) were installed parallel to the external plane of the leg $[22,23]$. It should be mentioned that the markers were fixed on the subjects' bodies with double-sided adhesive to prevent the markers' displacement. After performing clinical examinations and functional tests, the subjects ran in the determined path with their maximum speed and then performed cutting maneuvers in specified angles in predictable (dominant leg side) and unpredictable ways (determining the direction of movement with the lightening of white and blue colors), three times in each movement and a random order (so that several subjects first performed predictable maneuvers and some others first performed unpredictable maneuvers). Their movements were recorded and analyzed. The method of performing the cutting maneuver was that the person ran the distance of $10 \mathrm{~m}$ with ultimate speed and, after performing a pair jump to the adjusted ball (based on the amount of vertical jump of each person), she landed and by restarting towards the determined place to perform the cutting side moved and performed the maneuver at the specified angle (35- to 55-degree deviation from the main path) [24]. According to Figure 1, in a predictable cutting, the person goes towards the dominant leg. But according to Figure 2, in unpredictable cutting, by touching the range of $80 \%$ to $100 \%$ of the length of a step, before reaching the place of cutting, she decided the direction of movement through the lightened color. Thus, when the white color was on, the person performed the cutting side maneuver to the left side, and when the blue color was on, she performed the cutting side maneuver to the right side $[7,25,26]$.

Six rapture- $\mathrm{H}$ infrared cameras and the three-dimensional optoelectronic system of Motion Analysis (Motion Analysis Company, made in the USA) were used for the three-dimensional record of the subjects' performing cutting maneuvers. Depending on the type of skill, sampling was performed at a frequency of $200 \mathrm{~Hz}$. Also, the cameras were arranged so that on each plate, two cameras were placed at an angle of 45 to 60 degrees to each other so that at any moment, the information of each marker was recorded by at least two cameras. Finally, the obtained kinematic data were processed in MATLAB software, and then, using SPSS v. 22, the statistical analysis of the obtained data was performed. To compare the ratio of changes in the kinematic parameters of the knee during the predicted and unpredictable cutting side maneuver, we selected the mixed repeated measure ANOVA statistical method. But because of the unconformities of the homogeneity of covariance and Boxes test, the 1-way ANOVA test was used according to the literature [19], eliminating the possible disturbing effect of speed and agility between the subjects $(\mathrm{P}<0.05)$. It should be mentioned that before performing the mentioned test, the presuppositions of normality and randomness of data and the homogeneity of variance were confirmed by Shapiro-Wilk, Runs, and Levene's tests $(\mathrm{P}<0.05)$.

\section{Results}

In the present research, female adolescent athletes with a Mean \pm SD weight of $48.03 \pm 1.22 \mathrm{~kg}$, a height of $1.61 \pm 0.08 \mathrm{~m}$, and an age of $14.5 \pm 0.12 \mathrm{y}$ participated (Table 1). The Shapiro-Wilk and Runs tests showed that the parameters of flexion, valgus, and rotation of the leg in all four groups have a normal and random distribution $(\mathrm{P}<0.05)$; Levene's test results showed that the variance homogeneity presupposition was confirmed for all data. Therefore, a 1-way ANOVA test was used to compare the kinematic parameters in two predictable and unpredictable states between the ball and racket athletes $(\mathrm{P}<0.05)$. Also, to eliminate disturbing factors such as speed and agility, the results of the ANOVA test showed no significant difference between athletes in various fields regarding the ratio of speed $(\mathrm{P}=0.07)$ and agility $(\mathrm{P}=0.06)$.

In Table 1, the results of the statistical test indicate that the kinematic parameters of knee flexion $(\mathrm{P}=0.86)$, knee valgus $(\mathrm{P}=0.56)$, and tibia rotation $(\mathrm{P}=0.18)$, during the predicted cutting between the ball and racket athletes are not significant $(\mathrm{P}>0.05)$. But Table 2 shows that during unpredictable cutting side in the kinematic parameters of knee flexion $(\mathrm{P}=0.003)$, knee valgus $(\mathrm{P}=0.001)$, and tibia rotation $(\mathrm{P}=0.001)$, a significant difference was observed between the ball and racket athletes $(\mathrm{P}<0.05)$. In addition, the results of post hoc tests showed that these differences as intragroup are not significant in basketball, handball, badminton, and table tennis fields $(\mathrm{P}=1)$, but these values are significant between ball and racket sports fields $(\mathrm{P}=0.003)$. In Figure 3, the mean changes of the mentioned parameters have also been shown in different conditions and groups.

\section{Discussion}

Since exercise has always been associated with the risk of injury, researchers have always try to identify, modify, and eliminate dangerous movement patterns. Cutting side movement is a risk factor for ACL injury, which is frequently performed by athletes in numerous sports fields such as basketball, volleyball, handball, football, tennis, squash, badminton, and so on [4]. Therefore, numerous studies examined the mentioned movement from various aspects and in different groups. Despite the multiple ar- 


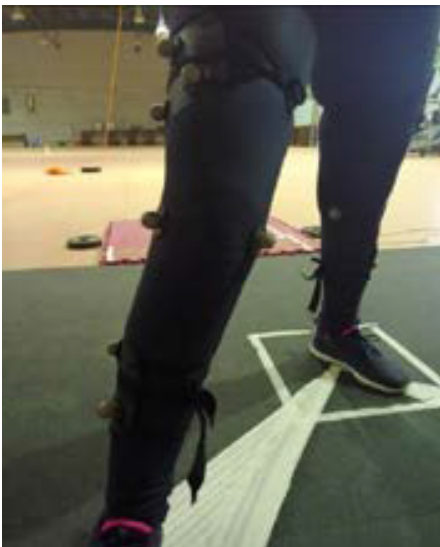

Figure 1. Predictable cutting side PHYSICAL TREATMENTS

ticles in identifying risk factors and preventing ACL injury, the authors did not find a study that examined the effect of predictability on cutting side movement patterns and changes in knee joint kinematic parameters between athletes in the ball and racket sports fields. Also, in the present research, we tried to test as close as possible to sports conditions; so that the person performed sprint, jumping, and landing before performing the cutting side of $10 \mathrm{~m}$ because most sports fields are performed in an open and unpredictable environment, and players may need to change direction and perform cutting movement after landing immediately. According to previous studies, the prevalence of knee injuries, especially ACL injury, is higher in women than men, and also adolescents are more exposed to injury than adults [26]. Therefore, considering the importance of injury prevention in this high-risk group, the present research investigated the effect of predictability on the knee kinematic parameters during the cutting side between the ball and racket athletes in Kerman Province, Iran.

The mechanism of ACL injury is often non-contact and is created following the load on the knee. If a person cannot control the musculoskeletal structure and correct the joint position for various reasons, the probability of injury increases by placing the knee joint in a position
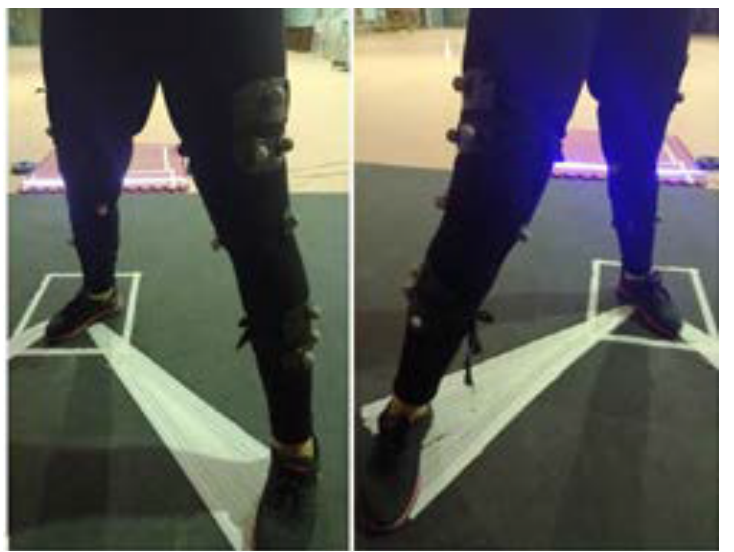

PHYSICAL TREA $\pitchfork$ MENTS

Figure 2. Unpredictable cutting side

of decreasing flexion and increasing valgus, and rotating the tibia. The mentioned kinematic parameters are performed in combination, and it is practically impossible to investigate one of them [15]. As it was observed in the results, the knee flexion parameter while performing unpredictable cutting side was significantly lower in ball athletes than in racket athletes, and also the knee valgus and tibia rotation parameters were significantly higher in ball athletes than in racket athletes.

According to literature, the anterior cruciate ligament is under high tension with less knee flexion during cutting movements. Thus, when the knee approaches the extension pattern, the pressure exerted on the ACL increases by the quadriceps muscles [27]. In this regard, Weir et al. showed that women have less knee flexion in cutting side movement than men, exposing them to ACL injury [28]. Yom et al. and Qu et al. also reported that during unpredictable cutting side, people showed less hip, trunk, and knee flexion, as well as more tibia rotation and valgus $[2,23]$. These results are consistent with the present research, but the rate of changes of these parameters was different, and this difference may be due to different-testing conditions and subjects. However, Kim et al. showed that unpredictable cutting maneuver leads to a significant

Table 1. The demographic characteristic of the study participants

\begin{tabular}{|c|c|c|c|c|}
\hline \multirow{2}{*}{ Groups } & \multicolumn{4}{|c|}{ Mean $\pm S D$} \\
\hline & Weight (kg) & Height (m) & Age (y) & Body Mass Index, $\mathrm{kg} / \mathrm{m}^{2}$ \\
\hline Badminton & $43.73 \pm 2.21$ & $1.58 \pm 1.99$ & $14.92 \pm 0.22$ & $17.02 \pm 0.56$ \\
\hline Table tennis & $49.13 \pm 2.46$ & $1.59 \pm 0.93$ & $14.69 \pm 0.286$ & $19.44 \pm 0.33$ \\
\hline Basketball & $51.65 \pm 2.03$ & $1.66 \pm 1.35$ & $14.50 \pm 0.314$ & $18.56 \pm 0.65$ \\
\hline Handball & $51.70 \pm 2.59$ & $1.63 \pm 1.74$ & $14.50 \pm 0.31$ & $19.23 \pm 0.44$ \\
\hline
\end{tabular}


Table 2. Kinematic parameters in predictable conditions

\begin{tabular}{|c|c|c|c|c|}
\hline Kinematic Parameters & Sports & Mean $\pm S D$ & $\mathbf{F}$ & $\mathbf{P}$ \\
\hline \multirow{5}{*}{ Knee flexion } & Badminton & $51.32 \pm 3.41$ & \multirow{5}{*}{0.24} & \multirow{5}{*}{0.86} \\
\hline & Table tennis & $50.98 \pm 3.21$ & & \\
\hline & & & & \\
\hline & Basketball & $50.93 \pm 2.88$ & & \\
\hline & Handball & $50.34 \pm 3.18$ & & \\
\hline \multirow{5}{*}{ Knee valgus } & Badminton & $15.75 \pm 0.75$ & \multirow{5}{*}{0.59} & \multirow{5}{*}{0.56} \\
\hline & Table tennis & $16.12 \pm 0.58$ & & \\
\hline & & & & \\
\hline & Basketball & $15.86 \pm 0.93$ & & \\
\hline & Handball & $16.23 \pm 1.12$ & & \\
\hline \multirow{5}{*}{ Tibia rotation } & Badminton & $6.68 \pm 1.14$ & \multirow{5}{*}{0.96} & \multirow{5}{*}{0.18} \\
\hline & Table tennis & $6.98 \pm 1.05$ & & \\
\hline & & & & \\
\hline & Basketball & $7.01 \pm 1.29$ & & \\
\hline & Handball & $7.12 \pm 1.11$ & & \\
\hline
\end{tabular}

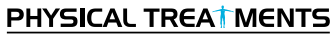

increase in knee flexion [29]. This research result is not consistent with the available results, and this difference can be due to the gender difference of the subjects.

Studies state that the valgus angle of the knee is one of the strongest predictors of ACL injury and has a direct correlation with the amount of abductor torque rate imposed on this joint. In this regard, Weir et al. examined the cutting maneuver in unpredictable conditions and stated that in these conditions, the valgus and varus knee torques are twice larger than performing the maneuver in the predicted conditions [30]. Despite the lack of studies

Table 3. Kinematic parameters in unpredictable conditions

\begin{tabular}{|c|c|c|c|c|}
\hline Kinematic Parameters & Sports & Mean $\pm S D$ & $\mathbf{F}$ & $\mathbf{P}$ \\
\hline \multirow{5}{*}{ Knee flexion } & Badminton & $49.42 \pm 3.17$ & & \\
\hline & Table tennis & $48.98 \pm 3.40$ & & \\
\hline & & & 5.53 & 0.003 \\
\hline & Basketball & $45.44 \pm 2.43$ & & \\
\hline & Handball & $44.89 \pm 3.33$ & & \\
\hline \multirow{5}{*}{ Knee valgus } & Badminton & $16.13 \pm 1.19$ & & \\
\hline & Table tennis & $15.99 \pm 1.15$ & & \\
\hline & & & 9.05 & 0.001 \\
\hline & Basketball & $17.78 \pm 1.22$ & & \\
\hline & Handball & $18.53 \pm 1.71$ & & \\
\hline \multirow{5}{*}{ Tibia rotation } & Badminton & $6.90 \pm 1.42$ & & \\
\hline & Table tennis & $7.06 \pm 1.16$ & & \\
\hline & & & 19.22 & 0.001 \\
\hline & Basketball & $9.75 \pm 1.21$ & & \\
\hline & Handball & $9.65 \pm 1.34$ & & \\
\hline
\end{tabular}




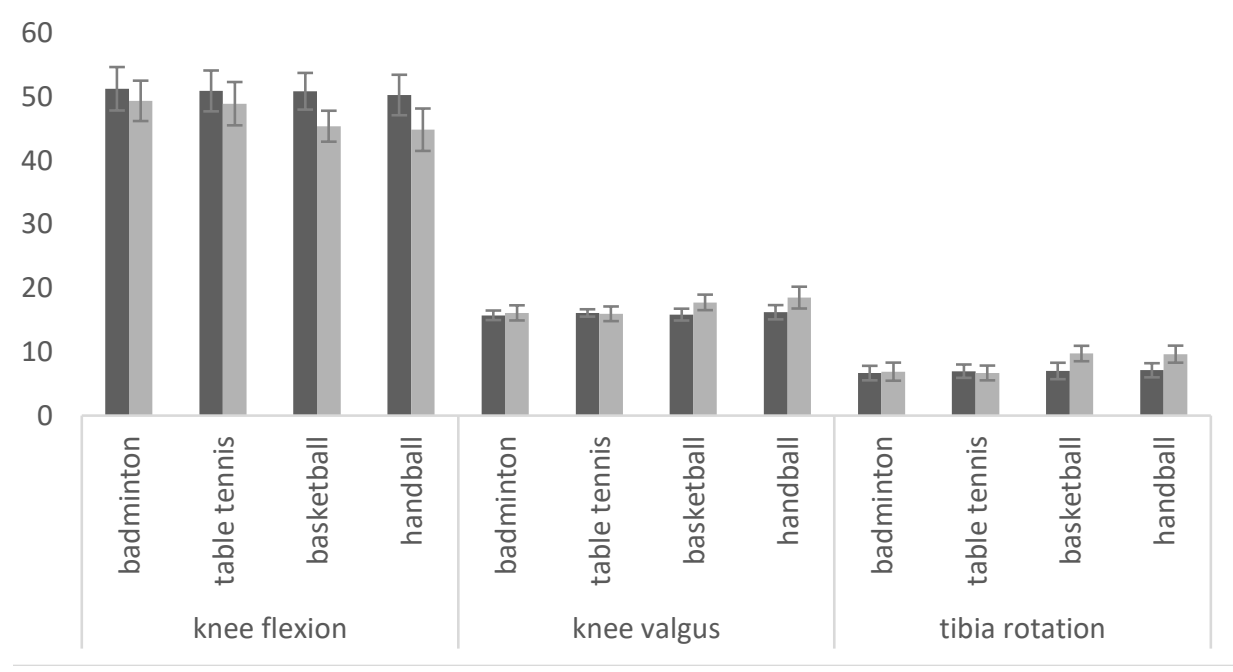

PHYSICAL TREA $\mid$ MENTS

Figure 3. Mean changes of the knee kinematic parameters in difference between ball and racket sports

in the field of three-dimensional kinematics and the report of the tibia rotation rate, some studies have reported anterior shear force as the primary determinant factor in the load rate imposed on ACL because this parameter has shown a significant correlation with the reduction ratio of knee flexion [31]. In this regard, Frendt et al. showed that male athletes had a significant difference in the ratio of kinematic parameters, including tibia rotation, while performing the predicted and unpredictable cutting maneuver [17]. The results of this study are consistent with the present research results. But the reported values are different; this difference has probably been due to the testing protocol and the different gender of the subjects.

According to previous studies, sports fields are different and can have various effects on athletes. For example, racket sports are among the reaction sports, in a way that table tennis and badminton players need not only a high level of physical function but also a variety of mental preparation and cognitive skills. Also, in competitive sports such as basketball, football and handball, and so on, players should receive and return a moving ball quickly, or even along the path cut the ball movement. To perform all these actions successfully, players should make the best predictions with the maximum speed and accuracy by precisely assessing the strengths and weaknesses of teammates and opponents $[1,32]$. Because of the lack of studies in the effects of perceptual abilities on kinematic parameters and movement pattern s and comparison of the ball and racket athletes, the existing studies are addressed in sports psychology and move- ment behavior. In sports psychology studies, Mirinejad et al. showed that perceptual and visual skills are more seen in racket athletes than ball athletes [33]. These results are consistent with the present rese arch in terms of better prediction ability in racket athletes. Based on these results, exercise factors related to sports fields can improve these perceptual abilities. Because of the effect of perceptual abilities such as predictability in kinematic parameters and correct execution of movement pattern, athletes and coaches of ball sports such as basketball and handball are recommended to strengthen perceptual factors such as predictability and reaction time in designing exercises, and also special attention should be paid to correct implementation of risky techniques. A limitation of the present study was overlooking psychological and nutritional conditions and the effects of reflective markers on participants' performance.

\section{Conclusion}

The present study results indicate more dangerous kinematic parameters in the group of ball sports such as basketball and handball. In other words, it was observed in the results that knee flexion was significantly lower in the ball sports group than in the racket sports, and also the parameters of knee valgus and tibia rotation were significantly higher in the ball sports group than in the racket sports. Adolescent girls are more exposed to the risk of injury, and this injury can have devastating effects on a person's life. So, it is suggested that to prevent sports injuries and psychological and physical consequences 
resulting from it, and to promote the level of competitions, the issue of predictability and correct training of techniques in designing exercises for this age group, especially in ball fields, receive more attention. Accordingly, the prevalence of non-contact injuries is reduced by increased athletes' awareness and correct performance.

\section{Ethical Considerations}

\section{Compliance with ethical guidelines}

All ethical principles were considered in this article. The participants were informed about the purpose of the research and its implementation sages; they were also assured about the confidentiality of their information; Moreover, They were allowed to leave the study whenever they wish, and if desired, the results of the research would be available to them. This study was approved by the Ethics Committee of Shahid Bahonar University of Kerman (Code: IR.UK.VETMED.REC.1398.022).

\section{Funding}

The present paper was extracted from the MSc. thesis of the first author at the Department of Sports Injuries and Corrective Exercises, Faculty of Sports Sciences, Shahid Bahonar University of Kerman.

\section{Authors' contributions}

Conceptualization, methodology, supervision: All authors; Invesigation, writing - original draft, funding acquisition, resources: Elham Hosseini; Writing - review and editing: Abdolhamid Daneshjoo, Mansour Sahebozaman.

\section{Conflict of interest}

The authors declared no conflict of interest.

\section{Acknowledgments}

The authors would like to thank Saeed Mollahosseini and Milad Fallahzadeh for their cooperation in the research process.

\section{References}

[1] Nuri L, Shadmehr A, Ghotbi N, Attarbashi Moghadam B. Reaction time and anticipatory skill of athletes in open and closed skill-dominated sport. European Journal of Sport Science. 2013; 13(5):431-6. [DOI:10.1080/17461391.2012.738712] [PMID]

[2] Yom JP, Owens T, Arnett S, Beebe J, Son V. The effects of an unanticipated side-cut on lower extremity kinematics and ground reaction forces during a drop landing. Sports Biomechanics. 2019; 18(4):414-25. [DOI:10.1080/14763141.2017.14097 95] [PMID]

[3] Hoffman J. Physiological aspects of sport training and performance. Champaign: Human Kinetics; 2014. https:// www.google.com/books/edition/Physiological_Aspects_ of_Sport_Training/HBwDAwAAQBAJ?hl=en\&gbpv=0

[4] Seyedi M, Rajabi R, Shirzad E, Zareei M. [Comparison of high-risk movement patterns of acl injury in male and female adolescent soccer players during cutting maneuver (Persian)]. Journal of Sport Medicine Review. 2016; 8(19):77-94 [DOI:10.1080/14763141.2017.1409795]

[5] Nhan DT, Klyce W, Lee RJ. Epidemiological patterns of alternative racket-sport injuries in the United States, 1997-2016. Orthopaedic Journal of Sports Medicine. 2018; 6(7):2325967118786237. [DOI:10.1177/2325967118786237] [PMID] [PMCID]

[6] Emami Hashemi SA, Rezvankhah Golsefidi N, Shirzad E, Mirkarimpour SH. [Kinematic comparison of anterior cruciate ligament injury risk between men and women during cutting maneuver. Razi Journal of Medical Sciences (Journal of Iran University of Medical Sciences). 2015; 22(138):12-22. https:/ / www.sid.ir/EN/JOURNAL/ViewPaper.aspx?ID=545891

[7] Mok KM, Bahr R, Krosshaug T. Reliability of lower limb biomechanics in two sport-specific side-step cutting tasks. Sports Biomechanics. 2018; 17(2):157-67. [DOI:10.1080/1476 3141.2016.1260766] [PMID]

[8] Hewett TE, Beynnon BD, Krosshaug T, Myklebust G. Preventing knee injuries. In Sports Injury Prevention (pp. 49-71). Hoboken, New Jersey: Wiley-Blackwell; 2009. [DOI:10.1002/9781444303612.ch5]

[9] Akhani PN, Gosai H, Mendpara S, Harsoda JM. Mental chronometry in table tennis players and football players: Who have faster reaction time. International Journal of Basic and Applied Physiology. 2015; 4(1):53-7. http://www.ijbap. com/upload/ijbap-2015/8-ijbap-2015.pdf

[10] Atan T, Akyol P. Reaction times of different branch athletes and correlation between reaction time parameters. Procedia-Social and Behavioral Sciences. 2014; 116:2886-9. [DOI:10.1016/j.sbspro.2014.01.674]

[11] Ak E, Koçak S. Coincidence-anticipation timing and reaction time in youth tennis and table tennis players. Perceptual and Motor Skills. 2010; 110(3 Pt 1):879-87. [DOI:10.2466/ pms.110.3.879-887] [PMID]

[12] Vidja KR, Bhabhor MK, Sarvaiya JL, Patel NS, Joshi V. Long term playing of badminton improves the visual reaction time. Age (years). 2015; 26:7-33. https://ijorim.com/siteadmin/article_issue/142804315218_Kalpesh_Physiology 
[13] Omar RH, Meng KY, Knight VF, Abd Manan F, Padri MNA. Visual anticipation time differences between athletes in open and closed skills sports. Malaysian Journal of Movement, Health \& Exercise. 2017; 6(1). [DOI:10.15282/ mohe.v6i1.80]

[14] Cochrane JL, Lloyd DG, Besier TF, Elliott BC, Doyle TL, Ackland TR. Training affects knee kinematics and kinetics in cutting maneuvers in sport. Medicine \& Science in Sports \& Exercise. 2010; 42(8):1535-44. [DOI:10.1249/ MSS.0b013e3181d03ba0] [PMID]

[15] Cortes N, Onate J, Morrison S. Differential effects of fatigue on movement variability. Gait \& Posture. 2014; 39(3):888-93. [DOI:10.1016/j.gaitpost.2013.11.020] [PMID] [PMCID]

[16] Ford KR, Myer GD, Toms HE, Hewett TE. Gender differences in the kinematics of unanticipated cutting in young athletes. Medicine and Science in Sports and Exercise. 2005; 37(1):124-9. [PMID]

[17] Frendt TR. Effects of Dual Tasking on Anticipated and Unanticipated Cutting Maneuvers on Knee Biomechanics in Collegiate Male Athletes [MSc. thesis]. Toledo: University of Toledo; 2017. https://etd.ohiolink.edu/apexprod/rws_olink/r/1501/10?clear=10\&p10_accession_ num=toledo1493290972920494

[18] Schneiders AG, Sullivan SJ, O'Malley KJ, Clarke SV, Knappstein SA, Taylor LJ. A valid and reliable clinical determination of footedness. PM \& R: The Journal of Injury, Function, and Rehabilitation. 2010; 2(9):835-41. [DOI:10.1016/j.pmrj.2010.06.004] [PMID]

[19] Pallant JF. SPSS survival manual: A step-by-step guide to data analysis with SPSS for Windows. New York, NY: McGrath Hill Google Scholar; 2007. https://www.semanticscholar.org/paper/SPSS-survival-manual-\%3A-astep-by-step-guide-to-data-Pallant/c85844729d8d2041f72f8668a45933610da237e2

[20] Brown SR. The relationship between leg dominance and knee mechanics during the cutting maneuver [MS. thesis]. Iniana: Indiana; 2012. https://cardinalscholar.bsu.edu/ handle/123456789/196127

[21] Winter DA. Biomechanics and motor control of human movement. New Jersey: John Wiley \& Sons; 2009. [DOI:10.1002/9780470549148]

[22] Dahaji AA, Amirseyfaddini M, Nikooie R. Comparison of selected lnee kinematic factors during single leg landing after six weeks of multi-angled isometric exercises on knee joint flexor and extensor muscle groups. Scientific Journal of Rehabilitation Medicine. 2019; 8(2):182-90. http:// www. medrehab.sbmu.ac.ir/article_1100616_866fdc73bdbc0e71f 1b04aa820a1a26e.pdf

[23] Sigward S, Powers CM. The influence of experience on knee mechanics during side-step cutting in females. Clinical Biomechanics. 2006; 21(7):740-7. [DOI:10.1016/j.clinbiomech.2006.03.003] [PMID]

[24] Qu X, Chen X. Combined effects of lower limb muscle fatigue and decision making to the knee joint during cutting maneuvers based on two different position-sense-acuity groups. Paper presented at: International Conference on Applied Human Factors and Ergonomics. $25-29$ July 1017; Cham, Vietnam. [DOI:10.1007/978-3-319-60822-8_13]
[25] Buermann S, Gloppen E, Kriechbaum R, Potter D, Sheehan N. Influence of fatigue and anticipation on knee kinematics and kinetics during a jump-cut maneuver. Sophia: Catherine University; 2017. https://sophia.stkate.edu/ dpt_papers/55/

[26] Hosseinzadeh S, Kiapour AM. Age-related changes in ACL morphology during skeletal growth and maturation are different between females and males. Journal of Orthopaedic Research. 2021; 39(4):841-9. [DOI:10.1002/jor.24748] [PMID]

[27] Yu B, Garrett WE. Mechanisms of non-contact ACL injuries. British Journal of Sports Medicine. 2007; 41(suppl 1):i47-51. [DOI:10.1136/bjsm.2007.037192] [PMID] [PMCID]

[28] Weir G, Stillman M, van Emmerik R, Wyatt H, Jewell C, Hamill J. Differences in kinetics, kinematics and muscle activation strategies in male and female team sport athletes during unanticipated sidestepping. Journal of Science in Sport and Exercise. 2019; 1(2):159-67. [DOI:10.1007/s42978-019-0019-2]

[29] Kim JH, Lee KK, Ahn KO, Kong SJ, Park SC, Lee YS. Evaluation of the interaction between contact force and decision making on lower extremity biomechanics during a side-cutting maneuver. Archives of Orthopaedic and Trauma Surgery. 2016; 136(6):821-8. [DOI:10.1007/s00402-016-2457-1] [PMID]

[30] Weir G, van Emmerik R, Jewell C, Hamill J. Coordination and variability during anticipated and unanticipated sidestepping. Gait \& Posture. 2019; 67:1-8. [DOI:10.1016/j.gaitpost.2018.09.007] [PMID]

[31] Hosseini E, Daneshjoo A, Sahebozamani M. Comparing the knee joint kinematic parameters of female athletes during sidestep cutting task before and after fatigue in predictable and unpredictable settings. Journal of Sport Biomechanics. 2019; 5(3):178-87. [DOI:10.32598/biomechanics.5.3.5]

[32] Hassani EN, Mohammadzadeh H. [The comparison of reaction time and coincidence-anticipation timing in elite and non-elite table tennis players (Persian)]. Sport Psychology. 2017; 2(2):55-64. https://mbsp.sbu.ac.ir/article_99661. html?lang=en

[33] Mirinezhad A, Ayoobi S, Fooladian J. [The comparison of selected visual skills of elite athletes in rocket and nonrocket sports (Persian)]. Sport Psychology. 2017; 2(2):1-12. https://mbsp.sbu.ac.ir/article_99626.html?lang=en 
This Page Intentionally Left Blank 